\title{
P, C and T: Different Properties on the Kinematical Level
}

\author{
Valeriy V. Dvoeglazov \\ UAF, Universidad Autónoma de Zacatecas \\ Ap. Postal 636, Suc. 3, Zacatecas 98061, Zac., México \\ E-mail: valeri@fisica.uaz.edu.mx
}

\begin{abstract}
We study the discrete symmetries $(P, C$ and $T)$ on the kinematical level within the extended Poincaré Group. On the basis of the Silagadze research, we investigate the question of the definitions of the discrete symmetry operators both on the classical level, and in the secondary-quantization scheme. We study the physical contents within several bases: light-front formulation, helicity basis, angular momentum basis, and so on, on several practical examples. We analize problems in construction of the neutral particles in the the $(1 / 2,0)+(0,1 / 2)$ representation, the $(1,0)+(0,1)$ and the $(1 / 2,1 / 2)$ representations of the Lorentz Group. As well known, the photon has the quantum numbers $1^{-}$, so the $(1,0)+(0,1)$ representation of the Lorentz group is relevant to its description. We have ambiguities in the definitions of the corresponding operators $P, C ; T$, which lead to different physical consequences. It appears that the answers are connected with the helicity basis properties, and commutations/anticommutations of the corresponding operators, $P, C, T$, and $C^{2}, P^{2},(C P)^{2}$ properties.
\end{abstract}

\section{Introduction.}

In his paper of 1992 Silagadze claimed: "It is shown that the usual situation when boson and its antiparticle have the same internal parity, while, fermion and its antiparticle have opposite parities, assumes a kind of locality 
of the theory. In general, when a quantum-mechanical parity operator is defined by means of the group extension technique, the reversed situation is also possible", Ref. [1]. Then, Ahluwalia et al proposed [5] the BargmannWightman-Wigner- type quantum field theory, where, as they claimed, the boson and the antiboson have oposite intrinsic parities (see also [6]). Actually, this type of theories has been first proposed by Gelfand and Tsetlin (1956), Ref. [7]. In fact, it is based on the two-dimensional representation of the inversion group. They indicated applicability of this theory to the description of the system of $K$-mesons and the possible relations to the LeeYang paper. The (anti)comutativity of the discrete symmetry operations has also been investigated by Foldy and Nigam [8], who claimed that it is related to the eigenvalues of the charge operator. The relations of the GelfandTsetlin construct to the representations of the anti-de Sitter $S O(3,2)$ group and the general relativity theory have also been discussed in subsequent papers of Sokolik. E. Wigner [9] presented some relevant results at the Istanbul School on Theoretical Physics in 1962. Later, Fushchich et al discussed the wave equations. Actually, the theory presented by Ahluwalia, Goldman and Johnson is the Dirac-like generalization of the Weinberg $2(2 J+1)$-theory for the spin 1. The equations have also been presented in the Sankaranarayanan and Good paper of 1965, Ref. [10]. In [11] the theory in the $\left(\frac{1}{2}, 0\right) \oplus\left(0, \frac{1}{2}\right)$ representation based on the chiral helicity 4-eigenspinors was proposed. The corresponding equations have been obtained, e. g., in [3]. However, later we found the papers by Ziino and Barut [12] and the Markov papers [13], which also have connections with the subject under consideration. The question of definitions of the discrete symmetries operators raised by Silagadze, has not yet been clarified in detail. Explicit examples are presented below and in the previous papers [2, 3, 4, 15, 6, 10, 11, 12, 13].

\section{Helicity Basis and Parity.}

The 4-spinors have been studied well when the basis has been chosen in such a way that they are eigenstates of the $\hat{\mathbf{S}}_{3}$ operator:

$$
u_{\frac{1}{2}, \frac{1}{2}}=N_{\frac{1}{2}}^{+}\left(\begin{array}{l}
1 \\
0 \\
1 \\
0
\end{array}\right), u_{\frac{1}{2},-\frac{1}{2}}=N_{-\frac{1}{2}}^{+}\left(\begin{array}{l}
0 \\
1 \\
0 \\
1
\end{array}\right)
$$




$$
v_{\frac{1}{2}, \frac{1}{2}}=N_{\frac{1}{2}}^{-}\left(\begin{array}{c}
1 \\
0 \\
-1 \\
0
\end{array}\right), v_{\frac{1}{2},-\frac{1}{2}}=N_{-\frac{1}{2}}^{-}\left(\begin{array}{c}
0 \\
1 \\
0 \\
-1
\end{array}\right) .
$$

And, oppositely, the helicity basis case has not been studied almost at all (see, however, Refs. [14, 15]). Let me remind that the boosted 4-spinors $\Psi(\mathbf{p})=\operatorname{column}\left(\phi_{R}(\mathbf{p}) \pm \phi_{L}(\mathbf{p})\right)$ in the common-used basis are the parity eigenstates with the eigenvalues of \pm 1 .

In the helicity spin basis the 2-eigenspinors of the helicity operator [16]

$$
\frac{1}{2} \sigma \cdot \widehat{\mathbf{p}}=\frac{1}{2}\left(\begin{array}{cc}
\cos \theta & \sin \theta e^{-i \phi} \\
\sin \theta e^{+i \phi} & -\cos \theta
\end{array}\right)
$$

$\theta, \phi$ are the angles of the spherical coordinate system, can be defined as follows [16, 17]:

$$
\phi_{\frac{1}{2} \uparrow} \sim\left(\begin{array}{c}
\cos \frac{\theta}{2} e^{-i \phi / 2} \\
\sin \frac{\theta}{2} e^{+i \phi / 2}
\end{array}\right), \quad \phi_{\frac{1}{2} \downarrow} \sim\left(\begin{array}{c}
\sin \frac{\theta}{2} e^{-i \phi / 2} \\
-\cos \frac{\theta}{2} e^{+i \phi / 2}
\end{array}\right),
$$

for $h= \pm 1 / 2=\uparrow \downarrow$ eigenvalues, respectively.

We start from the Klein-Gordon equation, generalized for describing the spin-1/2 particles (i. e., two degrees of freedom), $c=\hbar=1$ :

$$
(E+\sigma \cdot \mathbf{p})(E-\sigma \cdot \mathbf{p}) \phi=m^{2} \phi .
$$

It can be re-written in the form of the system of two first-order equations for 2 -spinors as in the Sakurai book. At the same time, we observe that they may be chosen as the eigenstates of the helicity operator:

$$
\begin{aligned}
& (E-(\sigma \cdot \mathbf{p})) \phi_{\uparrow}=(E-p) \phi_{\uparrow}=m \chi_{\uparrow}, \\
& (E+(\sigma \cdot \mathbf{p})) \chi_{\uparrow}=(E+p) \chi_{\uparrow}=m \phi_{\uparrow}, \\
& (E-(\sigma \cdot \mathbf{p})) \phi_{\downarrow}=(E+p) \phi_{\downarrow}=m \chi_{\downarrow}, \\
& (E+(\sigma \cdot \mathbf{p})) \chi_{\downarrow}=(E-p) \chi_{\downarrow}=m \phi_{\downarrow} .
\end{aligned}
$$

If the $\phi$ spinors are defined by the equation (44) then we can construct the 
corresponding $u-$ and $v-4$-spinors 1

$$
\begin{aligned}
& u_{\uparrow}=N_{\uparrow}^{+}\left(\begin{array}{c}
\phi_{\uparrow} \\
\frac{E-p}{m} \phi_{\uparrow}
\end{array}\right)=\frac{1}{\sqrt{2}}\left(\begin{array}{c}
\sqrt{\frac{E+p}{m}} \phi_{\uparrow} \\
\sqrt{\frac{m}{E+p}} \phi_{\uparrow}
\end{array}\right), \\
& u_{\downarrow}=N_{\downarrow}^{+}\left(\begin{array}{c}
\phi_{\downarrow} \\
\frac{E+p}{m} \phi_{\downarrow}
\end{array}\right)=\frac{1}{\sqrt{2}}\left(\begin{array}{c}
\sqrt{\frac{m}{E+p}} \phi_{\downarrow} \\
\sqrt{\frac{E+p}{m}} \phi_{\downarrow}
\end{array}\right), \\
& v_{\uparrow}=N_{\uparrow}^{-}\left(\begin{array}{c}
\phi_{\uparrow} \\
-\frac{E-p}{m} \phi_{\uparrow}
\end{array}\right)=\frac{1}{\sqrt{2}}\left(\begin{array}{c}
\sqrt{\frac{E+p}{m}} \phi_{\uparrow} \\
-\sqrt{\frac{m}{E+p}} \phi_{\uparrow}
\end{array}\right), \\
& v_{\downarrow}=N_{\downarrow}^{-}\left(\begin{array}{c}
\phi_{\downarrow} \\
-\frac{E+p}{m} \phi_{\downarrow}
\end{array}\right)=\frac{1}{\sqrt{2}}\left(\begin{array}{c}
\sqrt{\frac{m}{E+p}} \phi_{\downarrow} \\
-\sqrt{\frac{E+p}{m}} \phi_{\downarrow}
\end{array}\right),
\end{aligned}
$$

where the normalization to the unit $( \pm 1)$ was used. One can prove that the matrix $P=\gamma^{0}=\left(\begin{array}{ll}0 & I \\ I & 0\end{array}\right)$ can be used in the parity operator as in the original Dirac basis. Indeed, the 4-spinors (10],13) satisfy the Dirac equation in the spinorial (Weyl) representation of the $\gamma$-matrices. Hence, the parity-transformed function $\Psi^{\prime}(t,-\mathbf{x})=P \Psi(t, \mathbf{x})$ must satisfy $\left[i \gamma^{\mu} \partial_{\mu}^{\prime}-\right.$ $m] \Psi^{\prime}(t,-\mathbf{x})=0$ with $\partial_{\mu}^{\prime}=\left(\partial / \partial t,-\nabla_{i}\right)$. This is possible when $P^{-1} \gamma^{0} P=\gamma^{0}$ and $P^{-1} \gamma^{i} P=-\gamma^{i}$. The P-matrix above satisfies these requirements, as in the textbook case [18].

Next, it is easy to prove that one can form the projection operators

$$
\begin{aligned}
& \mathcal{P}_{+}=+\sum_{h} u_{h}(\mathbf{p}) \bar{u}_{h}(\mathbf{p})=\frac{p_{\mu} \gamma^{\mu}+m}{2 m} \\
& \mathcal{P}_{-}=-\sum_{h} v_{h}(\mathbf{p}) \bar{v}_{h}(\mathbf{p})=\frac{m-p_{\mu} \gamma^{\mu}}{2 m}
\end{aligned}
$$

with the properties $P_{+}+P_{-}=1$ and $P_{ \pm}^{2}=P_{ \pm}$. This permits us to expand the 4-spinors defined in the basis (1,2) in the linear superpositions of the helicity basis 4-spinors, and to find corresponding coefficients of the expansion:

$$
u_{\sigma}(\mathbf{p})=A_{\sigma h} u_{h}(\mathbf{p})+B_{\sigma h} v_{h}(\mathbf{p}), v_{\sigma}(\mathbf{p})=C_{\sigma h} u_{h}(\mathbf{p})+D_{\sigma h} v_{h}(\mathbf{p}) .
$$

\footnotetext{
${ }^{1}$ One can also try to construct yet another theory differing from the ordinary Dirac theory. The 4 -spinors may be not the eigenspinors of the helicity operator of the $(1 / 2,0) \oplus$ $(0,1 / 2)$ representation space, $c f$. [11]. They might be the eigenstates of the chiral helicity operator introduced in [11.
} 
Multiplying the above equations by $\bar{u}_{h^{\prime}}, \bar{v}_{h^{\prime}}$ respectively, and using the normalization conditions, we obtain $A_{\sigma h}=D_{\sigma h}=\bar{u}_{h} u_{\sigma}, B_{\sigma h}=C_{\sigma h}=-\bar{v}_{h} u_{\sigma}$. Thus, the transformation matrix from the common-used basis to the helicity basis is

$$
\left(\begin{array}{l}
u_{\sigma} \\
v_{\sigma}
\end{array}\right)=\mathcal{U}\left(\begin{array}{l}
u_{h} \\
v_{h}
\end{array}\right), \quad \mathcal{U}=\left(\begin{array}{ll}
A & B \\
B & A
\end{array}\right)
$$

Neither $A$ nor $B$ are unitary:

$$
\begin{aligned}
& A=\left(a_{++}+a_{+-}\right)\left(\sigma_{\mu} a^{\mu}\right)+\left(-a_{-+}+a_{--}\right)\left(\sigma_{\mu} a^{\mu}\right) \sigma_{3}, \\
& B=\left(-a_{++}+a_{+-}\right)\left(\sigma_{\mu} a^{\mu}\right)+\left(a_{-+}+a_{--}\right)\left(\sigma_{\mu} a^{\mu}\right) \sigma_{3},
\end{aligned}
$$

where

$$
\begin{aligned}
& a^{0}=-i \cos (\theta / 2) \sin (\phi / 2) \in \Im m, a^{1}=\sin (\theta / 2) \cos (\phi / 2) \in \Re e, \\
& a^{2}=\sin (\theta / 2) \sin (\phi / 2) \in \Re e, a^{3}=\cos (\theta / 2) \cos (\phi / 2) \in \Re e,
\end{aligned}
$$

and

$$
\begin{aligned}
& a_{++}=\frac{\sqrt{(E+m)(E+p)}}{2 \sqrt{2} m}, a_{+-}=\frac{\sqrt{(E+m)(E-p)}}{2 \sqrt{2} m}, \\
& a_{-+}=\frac{\sqrt{(E-m)(E+p)}}{2 \sqrt{2} m}, a_{--}=\frac{\sqrt{(E-m)(E-p)}}{2 \sqrt{2} m} .
\end{aligned}
$$

However, $A^{\dagger} A+B^{\dagger} B=I$, so the matrix $\mathcal{U}$ is unitary. Please note that this matrix acts on the spin indices $(\sigma, h)$, and not on the spinorial indices; it is $4 \times 4$ matrix.

We now investigate the properties of the helicity-basis 4-spinors with respect to the discrete symmetry operations $P, C$ and $T$. It is expected that $h \rightarrow-h$ under parity, as Berestetskiu, Lifshitz and Pitaevskiu claimed [19]. Indeed, if $\mathbf{x} \rightarrow-\mathbf{x}$, then the vector $\mathbf{p} \rightarrow-\mathbf{p}$, but the axial vector $\mathbf{S} \rightarrow$ $\mathbf{S}$, that implies the above statement. The helicity 2-eigenspinors transform $\phi_{\uparrow \downarrow} \Rightarrow-i \phi_{\downarrow \uparrow}$ with respect to $\mathbf{p} \rightarrow-\mathbf{p}$, Ref. [17]. Hence,

$$
\begin{aligned}
& P u_{\uparrow}(-\mathbf{p})=-i u_{\downarrow}(\mathbf{p}), P v_{\uparrow}(-\mathbf{p})=+i v_{\downarrow}(\mathbf{p}), \\
& P u_{\downarrow}(-\mathbf{p})=-i u_{\uparrow}(\mathbf{p}), P v_{\downarrow}(-\mathbf{p})=+i v_{\uparrow}(\mathbf{p}) .
\end{aligned}
$$

Thus, on the level of classical fields, we observe that the helicity 4-spinors transform to the 4-spinors of the opposite helicity. 
Also,

$$
\begin{aligned}
& C u_{\uparrow}(\mathbf{p})=-v_{\downarrow}(\mathbf{p}), C v_{\uparrow}(\mathbf{p})=+u_{\downarrow}(\mathbf{p}), \\
& C u_{\downarrow}(\mathbf{p})=+v_{\uparrow}(\mathbf{p}), C v_{\downarrow}(\mathbf{p})=-u_{\uparrow}(\mathbf{p}) .
\end{aligned}
$$

due to the properties of the Wigner operator $\Theta \phi_{\uparrow}^{*}=-\phi_{\downarrow}$ and $\Theta \phi_{\downarrow}^{*}=+\phi_{\uparrow}$, $\Theta_{[1 / 2]}=-i \sigma_{2}$. Similar conclusions can be obtained in the Fock space.

We define the field operator as follows:

$$
\Psi\left(x^{\mu}\right)=\sum_{h} \int \frac{d^{3} \mathbf{p}}{(2 \pi)^{3}} \frac{\sqrt{m}}{2 E}\left[u_{h}(\mathbf{p}) a_{h}(\mathbf{p}) e^{-i p_{\mu} x^{\mu}}+v_{h}(\mathbf{p}) b_{h}^{\dagger}(\mathbf{p}) e^{+i p_{\mu} x^{\mu}}\right] .
$$

The commutation relations are assumed to be the standard ones [21, 22, 18, 20] (compare with Refs. [3, 11]). If one defines $U_{P} \Psi\left(x^{\mu}\right) U_{P}^{-1}=\gamma^{0} \Psi\left(x^{\mu^{\prime}}\right)$, $U_{C} \Psi\left(x^{\mu}\right) U_{C}^{-1}=C \Psi^{\dagger}\left(x^{\mu}\right)$ and the anti-unitary operator of time reversal $\left(V_{T} \Psi\left(x^{\mu}\right) V_{T}^{-1}\right)^{\dagger}=T \Psi^{\dagger}\left(x^{\mu^{\prime \prime}}\right)$, then it is easy to obtain the corresponding transformations of the creation/annihilation operators:

$$
\begin{aligned}
& U_{P} a_{h}(\mathbf{p}) U_{P}^{-1}=-i a_{-h}(-\mathbf{p}), \quad U_{P} b_{h}(\mathbf{p}) U_{P}^{-1}=-i b_{-h}(-\mathbf{p}), \\
& U_{C} a_{h}(\mathbf{p}) U_{C}^{-1}=(-1)^{\frac{1}{2}+h} b_{-h}(\mathbf{p}), \quad U_{C} b_{h}(\mathbf{p}) U_{C}^{-1}=(-1)^{\frac{1}{2}-h} a_{-h}(-\mathbf{p}) .
\end{aligned}
$$

As a consequence, we obtain (provided that $U_{P}|0>=| 0>, U_{C}|0>=| 0>$ )

$$
\begin{aligned}
& U_{P} a_{h}^{\dagger}(\mathbf{p})\left|0>=U_{P} a_{h}^{\dagger} U_{P}^{-1}\right| 0>=i a_{-h}^{\dagger}(-\mathbf{p})|0>=i|-\mathbf{p},-h>^{+}, \\
& U_{P} b_{h}^{\dagger}(\mathbf{p})\left|0>=U_{P} b_{h}^{\dagger} U_{P}^{-1}\right| 0>=i b_{-h}^{\dagger}(-\mathbf{p})|0>=i|-\mathbf{p},-h>^{-},
\end{aligned}
$$

and

$$
\begin{aligned}
& U_{C} a_{h}^{\dagger}(\mathbf{p})\left|0>=U_{C} a_{h}^{\dagger} U_{C}^{-1}\right| 0>=(-1)^{\frac{1}{2}+h} b_{-h}^{\dagger}(\mathbf{p}) \mid 0>= \\
& (-1)^{\frac{1}{2}+h} \mid \mathbf{p},-h>^{-}, \\
& U_{C} b_{h}^{\dagger}(\mathbf{p})\left|0>=U_{C} b_{h}^{\dagger} U_{C}^{-1}\right| 0>=(-1)^{\frac{1}{2}-h} a_{-h}^{\dagger}(\mathbf{p}) \mid 0>= \\
& (-1)^{\frac{1}{2}-h} \mid \mathbf{p},-h>^{+} .
\end{aligned}
$$

Finally, for the $C P$ operation one should obtain:

$$
U_{P} U_{C} a_{h}^{\dagger}(\mathbf{p})\left|0>=-U_{C} U_{P} a_{h}^{\dagger}(\mathbf{p})\right| 0>=(-1)^{\frac{1}{2}+h} U_{P} b_{-h}^{\dagger}(\mathbf{p}) \mid 0>=
$$




$$
\begin{aligned}
= & i(-1)^{\frac{1}{2}+h} b_{h}^{\dagger}(-\mathbf{p})\left|0>=i(-1)^{\frac{1}{2}+h}\right|-\mathbf{p}, h>^{-}, \\
& U_{P} U_{C} b_{h}^{\dagger}(\mathbf{p})\left|0>=-U_{C} U_{P} b_{h}^{\dagger}(\mathbf{p})=(-1)^{\frac{1}{2}-h} U_{P} a_{-h}^{\dagger}(\mathbf{p})\right| 0>= \\
= & i(-1)^{\frac{1}{2}-h} a_{h}^{\dagger}(-\mathbf{p})\left|0>=i(-1)^{\frac{1}{2}-h}\right|-\mathbf{p}, h>^{+} .
\end{aligned}
$$

As in the classical case, the $P$ and $C$ operations anticommute in the $\left(\frac{1}{2}, 0\right) \oplus$ $\left(0, \frac{1}{2}\right)$ quantized case. This opposes to the theory based on the 4-spinor eigenstates of chiral helicity (cf. [3, 8]). Since the $V_{T}$ is an anti-unitary operator the problem must be solved after taking into account of the fact that the $c$-numbers should be put outside the hermitian conjugation without complex conjugation:

$$
\left[V_{T} h A V_{T}^{-1}\right]^{\dagger}=\left[h^{*} V_{T} A V_{T}^{-1}\right]^{\dagger}=h\left[V_{T} A^{\dagger} V_{T}^{-1}\right] .
$$

After applying this definition we obtain:2

$$
\begin{aligned}
& V_{T} a_{h}^{\dagger}(\mathbf{p}) V_{T}^{-1}=+i(-1)^{\frac{1}{2}-h} a_{h}^{\dagger}(-\mathbf{p}) \\
& V_{T} b_{h}(\mathbf{p}) V_{T}^{-1}=+i(-1)^{\frac{1}{2}-h} b_{h}(-\mathbf{p})
\end{aligned}
$$

Furthermore, we observed that the parity properties depend on the phase factor in the following definition:

$$
U_{P} \Psi(t, \mathbf{x}) U_{P}^{-1}=e^{i \alpha} \gamma^{0} \Psi(t,-\mathbf{x}) .
$$

Indeed,

$$
\begin{aligned}
& U_{P} a_{h}(\mathbf{p}) U_{P}^{-1}=-i e^{i \alpha} a_{-h}(-\mathbf{p}) \\
& U_{P} b_{h}^{\dagger}(\mathbf{p}) U_{P}^{-1}=+i e^{i \alpha} b_{-h}^{\dagger}(-\mathbf{p})
\end{aligned}
$$

From this, if $\alpha=\pi / 2$ we obtain opposite parity properties of the creation/annihilation operators for particles and anti-particles:

$$
\begin{aligned}
& U_{P} a_{h}(\mathbf{p}) U_{P}^{-1}=+a_{-h}(-\mathbf{p}), \\
& U_{P} b_{h}(\mathbf{p}) U_{P}^{-1}=-b_{-h}(-\mathbf{p}) .
\end{aligned}
$$

However, the difference with the Dirac case still preserves ( $h$ transforms to $-h)$. We find similar situation with the question of constructing the neutrino field operator (cf. with the Goldhaber-Kayser creation phase factor).

\footnotetext{
${ }^{2} T$ should be chosen in such a way in order to fulfill $T^{-1} \gamma_{0}^{T} T=\gamma_{0}, T^{-1} \gamma_{i}^{T} T=\gamma_{i}$ and $T^{T}=-T$, as in Ref. [21].
} 
Next, we find the explicit form of the parity operator $U_{P}$ and prove that it commutes with the Hamiltonian operator. We prefer to use the method described in [20, $\S 10.2-10.3]$. It is based on the anzatz that $U_{P}=$ $\exp [i \alpha \hat{A}] \exp [i \hat{B}] \quad$ with $\hat{A}=\sum_{s} \int d^{3} \mathbf{p}\left[a_{\mathbf{p}, s}^{\dagger} a_{-\mathbf{p} s}+b_{\mathbf{p} s}^{\dagger} b_{-\mathbf{p} s}\right]$ and $\hat{B}=\sum_{s} \int d^{3} \mathbf{p}\left[\beta a_{\mathbf{p}, s}^{\dagger} a_{\mathbf{p} s}+\gamma b_{\mathbf{p} s}^{\dagger} b_{\mathbf{p} s}\right]$. On using the known operator identity

$$
e^{\hat{A}} \hat{B} e^{-\hat{A}}=\hat{B}+[\hat{A}, \hat{B}]_{-}+\frac{1}{2 !}[\hat{A},[\hat{A}, \hat{B}]]+\ldots
$$

and $[\hat{A}, \hat{B} \hat{C}]_{-}=[\hat{A}, \hat{B}]_{+} \hat{C}-\hat{B}[\hat{A}, \hat{C}]_{+}$one can fix the parameters $\alpha, \beta, \gamma$ such that one satisfies the physical requirements that a Dirac particle and its anti-particle have opposite intrinsic parities.

In our case, we need to satisfy the requirement that the operator should invert not only the sign of the momentum, but the sign of the helicity too. We may achieve this goal by the analogous postulate $U_{P}=e^{i \alpha \hat{A}}$ with

$$
\hat{A}=\sum_{h} \int \frac{d^{3} \mathbf{p}}{2 E}\left[a_{h}^{\dagger}(\mathbf{p}) a_{-h}(-\mathbf{p})+b_{h}^{\dagger}(\mathbf{p}) b_{-h}(-\mathbf{p})\right] .
$$

By direct verification, the requirement is satisfied provided that $\alpha=\pi / 2$. You may compare this parity operator with that given in [18, 20] for Dirac fields $\sqrt[3]{3}$

$$
\begin{aligned}
U_{P} & =\exp \left[i \frac { \pi } { 2 } \int d ^ { 3 } \mathbf { p } \sum _ { s } \left(a(\mathbf{p}, s)^{\dagger} a(\tilde{\mathbf{p}}, s)+b(\mathbf{p}, s)^{\dagger} b(\tilde{\mathbf{p}}, s)-\right.\right. \\
& \left.\left.-a(\mathbf{p}, s)^{\dagger} a(\mathbf{p}, s)+b(\mathbf{p}, s)^{\dagger} b(\mathbf{p}, s)\right)\right], \quad(10.69) \text { of }[20] .
\end{aligned}
$$

By direct verification one can also come to the conclusion that our new $U_{P}$ commutes with the Hamiltonian:

$$
\mathcal{H}=\int d^{3} \mathbf{x} \Theta^{00}=\int d^{3} \mathbf{k} \sum_{h}\left[a_{h}^{\dagger}(\mathbf{k}) a_{h}(\mathbf{k})-b_{h}(\mathbf{k}) b_{h}^{\dagger}(\mathbf{k})\right],
$$

i.e. $\left[U_{P}, \mathcal{H}\right]_{-}=0$. Alternatively, we can try to choose other commutation relations [3, 11] for the set of bi-orthonormal states. The formulas of the

\footnotetext{
${ }^{3}$ Greiner used the following commutation relations $\left[a(\mathbf{p}, s), a^{\dagger}\left(\mathbf{p}^{\prime}, s^{\prime}\right)\right]_{+}=$ $\left[b(\mathbf{p}, s), b^{\dagger}\left(\mathbf{p}^{\prime}, s^{\prime}\right)\right]_{+}=\delta^{3}\left(\mathbf{p}-\mathbf{p}^{\prime}\right) \delta_{s s^{\prime}}$. One should also note that the Greiner form of the parity operator is not the unique one. Itzykson and Zuber [18] proposed another one differing by the phase factors from (10.69) of [20]. In order to find relations between those two forms of the parity operator one should apply additional rotation in the Fock space.
} 
theory have been presented in the $\left(\frac{1}{2}, 0\right) \oplus\left(0, \frac{1}{2}\right)$ representation based on the chiral helicity 4-eigenspinors, see below. Next, the theory, which is based on a system of 6 -component Weinberg-like equations in the $(1,0) \oplus(0,1)$ representation, has also been constructed. The results are similar. The papers by Ziino and Barut [12] and the Markov papers [13] have connections with the subject under consideration.

\section{Chiral Helicity Construct and the Different Definition of the Charge Conjugate Oper- ator on the Secondary Quantization Level.}

In the chiral representation one can choose the spinorial basis (zero-momentum spinors) in the following way:

$$
\begin{aligned}
& \lambda_{\uparrow}^{S}(\mathbf{0})=\sqrt{\frac{m}{2}}\left(\begin{array}{c}
0 \\
i \\
1 \\
0
\end{array}\right), \lambda_{\downarrow}^{S}(\mathbf{0})=\sqrt{\frac{m}{2}}\left(\begin{array}{c}
-i \\
0 \\
0 \\
1
\end{array}\right), \\
& \lambda_{\uparrow}^{A}(\mathbf{0})=\sqrt{\frac{m}{2}}\left(\begin{array}{c}
0 \\
-i \\
1 \\
0
\end{array}\right), \lambda_{\downarrow}^{A}(\mathbf{0})=\sqrt{\frac{m}{2}}\left(\begin{array}{c}
i \\
0 \\
0 \\
1
\end{array}\right), \\
& \rho_{\uparrow}^{S}(\mathbf{0})=\sqrt{\frac{m}{2}}\left(\begin{array}{c}
1 \\
0 \\
0 \\
-i
\end{array}\right), \rho_{\downarrow}^{S}(\mathbf{0})=\sqrt{\frac{m}{2}}\left(\begin{array}{c}
0 \\
1 \\
i \\
0
\end{array}\right), \\
& \rho_{\uparrow}^{A}(\mathbf{0})=\sqrt{\frac{m}{2}}\left(\begin{array}{c}
1 \\
0 \\
0 \\
i
\end{array}\right), \rho_{\downarrow}^{A}(\mathbf{0})=\sqrt{\frac{m}{2}}\left(\begin{array}{c}
0 \\
1 \\
-i \\
0
\end{array}\right) .
\end{aligned}
$$

The indices $\uparrow \downarrow$ should be referred to the chiral helicity quantum number introduced in Ref. [11], $\eta=-h \gamma_{5}$. We use the notation of the previous papers on the subject. Ahluwalia and Grumiller used the helicity basis for

the 2nd-type 4-spinors. The reader would immediately find the 4-spinors of 
the second kind $\lambda_{\uparrow \downarrow}^{S, A}\left(p^{\mu}\right)$ and $\rho_{\uparrow \downarrow}^{S, A}\left(p^{\mu}\right)$ in an arbitrary frame on using the boost operators:

$$
\begin{gathered}
\lambda_{\uparrow}^{S}\left(p^{\mu}\right)=\frac{1}{2 \sqrt{E+m}}\left(\begin{array}{c}
i p_{l} \\
i\left(p^{-}+m\right) \\
p^{-}+m \\
-p_{r}
\end{array}\right), \lambda_{\downarrow}^{S}\left(p^{\mu}\right)=\frac{1}{2 \sqrt{E+m}}\left(\begin{array}{c}
-i\left(p^{+}+m\right) \\
-i p_{r} \\
-p_{l} \\
\left(p^{+}+m\right)
\end{array}\right), \\
\lambda_{\uparrow}^{A}\left(p^{\mu}\right)=\frac{1}{2 \sqrt{E+m}}\left(\begin{array}{c}
-i p_{l} \\
\left(p^{-}+m\right) \\
-p_{r}
\end{array}\right), \lambda_{\downarrow}^{A}\left(p^{\mu}\right)=\frac{1}{2 \sqrt{E+m}}\left(\begin{array}{c}
i\left(p^{+}+m\right) \\
i p_{r} \\
-p_{l} \\
\left(p^{+}+m\right)
\end{array}\right),
\end{gathered}
$$

and

$$
\begin{gathered}
\rho_{\uparrow}^{S}\left(p^{\mu}\right)=\frac{1}{2 \sqrt{E+m}}\left(\begin{array}{c}
p^{+}+m \\
p_{r} \\
i p_{l} \\
-i\left(p^{+}+m\right)
\end{array}\right), \rho_{\downarrow}^{S}\left(p^{\mu}\right)=\frac{1}{2 \sqrt{E+m}}\left(\begin{array}{c}
p_{l} \\
\left(p^{-}+m\right) \\
i\left(p^{-}+m\right) \\
-i p_{r}
\end{array}\right), \\
\rho_{\uparrow}^{A}\left(p^{\mu}\right)=\frac{1}{2 \sqrt{E+m}}\left(\begin{array}{c}
p^{+}+m \\
p_{r} \\
-i p_{l} \\
i\left(p^{+}+m\right)
\end{array}\right), \rho_{\downarrow}^{A}\left(p^{\mu}\right)=\frac{1}{2 \sqrt{E+m}}\left(\begin{array}{c}
\left(p^{-}+m\right) \\
-i\left(p^{-}+m\right) \\
i p_{r}
\end{array}\right) .
\end{gathered}
$$

Some of the 4-spinors are connected each other. The normalization of the spinors $\lambda_{\uparrow \downarrow}^{S, A}\left(p^{\mu}\right)$ and $\rho_{\uparrow \downarrow}^{S, A}\left(p^{\mu}\right)$ are the following ones:

$$
\begin{aligned}
& \bar{\lambda}_{\uparrow}^{S}\left(p^{\mu}\right) \lambda_{\downarrow}^{S}\left(p^{\mu}\right)=-i m, \quad \bar{\lambda}_{\downarrow}^{S}\left(p^{\mu}\right) \lambda_{\uparrow}^{S}\left(p^{\mu}\right)=+i m, \\
& \bar{\lambda}_{\uparrow}^{A}\left(p^{\mu}\right) \lambda_{\downarrow}^{A}\left(p^{\mu}\right)=+i m, \quad \bar{\lambda}_{\downarrow}^{A}\left(p^{\mu}\right) \lambda_{\uparrow}^{A}\left(p^{\mu}\right)=-i m, \\
& \bar{\rho}_{\uparrow}^{S}\left(p^{\mu}\right) \rho_{\downarrow}^{S}\left(p^{\mu}\right)=+i m \quad, \quad \bar{\rho}_{\downarrow}^{S}\left(p^{\mu}\right) \rho_{\uparrow}^{S}\left(p^{\mu}\right)=-i m, \\
& \bar{\rho}_{\uparrow}^{A}\left(p^{\mu}\right) \rho_{\downarrow}^{A}\left(p^{\mu}\right)=-i m, \quad \bar{\rho}_{\downarrow}^{A}\left(p^{\mu}\right) \rho_{\uparrow}^{A}\left(p^{\mu}\right)=+i m,
\end{aligned}
$$

All other conditions are equal to zero.

Implying that $\lambda^{S}\left(p^{\mu}\right)$ (and $\rho^{A}\left(p^{\mu}\right)$ ) answer for positive-frequency solutions; $\lambda^{A}\left(p^{\mu}\right)$ (and $\rho^{S}\left(p^{\mu}\right)$ ), for negative-frequency solutions, one can deduce the dynamical coordinate-space equations [3]:

$$
\begin{aligned}
& i \gamma^{\mu} \partial_{\mu} \lambda^{S}(x)-m \rho^{A}(x)=0, \\
& i \gamma^{\mu} \partial_{\mu} \rho^{A}(x)-m \lambda^{S}(x)=0, \\
& i \gamma^{\mu} \partial_{\mu} \lambda^{A}(x)+m \rho^{S}(x)=0, \\
& i \gamma^{\mu} \partial_{\mu} \rho^{S}(x)+m \lambda^{A}(x)=0 .
\end{aligned}
$$


They can be written in the 8-component form. This is just another representation of the Dirac-like equation in the appropriate Clifford Algebra. One can also re-write the equations into the two-component form, the Feynman-GellMann equations. In the Fock space the operators of the charge conjugation and space inversions can be defined as in the previous Section. We imply the bi-orthonormal system of the anticommutation relations. As a result we have the following properties of the creation (annihilation) operators in the Fock space:

$$
\begin{aligned}
& U_{[1 / 2]}^{s} a_{\uparrow}(\mathbf{p})\left(U_{[1 / 2]}^{s}\right)^{-1}=-i a_{\downarrow}(-\mathbf{p}), U_{[1 / 2]}^{s} a_{\downarrow}(\mathbf{p})\left(U_{[1 / 2]}^{s}\right)^{-1}=+i a_{\uparrow}(-\mathbf{p})(64) \\
& U_{[1 / 2]}^{s} b_{\uparrow}^{\dagger}(\mathbf{p})\left(U_{[1 / 2]}^{s}\right)^{-1}=+i b_{\downarrow}^{\dagger}(-\mathbf{p}), U_{[1 / 2]}^{s} b_{\downarrow}^{\dagger}(\mathbf{p})\left(U_{[1 / 2]}^{s}\right)^{-1}=-i b_{\uparrow}(-\mathbf{p}),(65)
\end{aligned}
$$

that signifies that the states created by the operators $a^{\dagger}(\mathbf{p})$ and $b^{\dagger}(\mathbf{p})$ have different properties with respect to the space inversion operation, comparing with Dirac states (the case also regarded in [12]):

$$
\begin{aligned}
& U_{[1 / 2]}^{s}\left|\mathbf{p}, \uparrow>^{+}=+i\right|-\mathbf{p}, \downarrow>^{+}, U_{[1 / 2]}^{s}\left|\mathbf{p}, \uparrow>^{-}=+i\right|-\mathbf{p}, \downarrow>^{-}, \\
& U_{[1 / 2]}^{s}\left|\mathbf{p}, \downarrow>^{+}=-i\right|-\mathbf{p}, \uparrow>^{+}, U_{[1 / 2]}^{s}\left|\mathbf{p}, \downarrow>^{-}=-i\right|-\mathbf{p}, \uparrow>^{-} .
\end{aligned}
$$

For the charge conjugation operation in the Fock space we have two physically different possibilities. The first one, e.g.,

$$
\begin{aligned}
& U_{[1 / 2]}^{c} a_{\uparrow}(\mathbf{p})\left(U_{[1 / 2]}^{c}\right)^{-1}=+b_{\uparrow}(\mathbf{p}), U_{[1 / 2]}^{c} a_{\downarrow}(\mathbf{p})\left(U_{[1 / 2]}^{c}\right)^{-1}=+b_{\downarrow}(\mathbf{p}), \\
& U_{[1 / 2]}^{c} b_{\uparrow}^{\dagger}(\mathbf{p})\left(U_{[1 / 2]}^{c}\right)^{-1}=-a_{\uparrow}^{\dagger}(\mathbf{p}), U_{[1 / 2]}^{c} b_{\downarrow}^{\dagger}(\mathbf{p})\left(U_{[1 / 2]}^{c}\right)^{-1}=-a_{\downarrow}^{\dagger}(\mathbf{p}),
\end{aligned}
$$

in fact, has some similarities with the Dirac construct. The action of this operator on the physical states are

$$
\begin{aligned}
& U_{[1 / 2]}^{c}\left|\mathbf{p}, \uparrow>^{+}=+\right| \mathbf{p}, \uparrow>^{-}, U_{[1 / 2]}^{c}\left|\mathbf{p}, \downarrow>^{+}=+\right| \mathbf{p}, \downarrow>^{-}, \\
& U_{[1 / 2]}^{c}\left|\mathbf{p}, \uparrow>^{-}=-\right| \mathbf{p}, \uparrow>^{+}, U_{[1 / 2]}^{c}\left|\mathbf{p}, \downarrow>^{-}=-\right| \mathbf{p}, \downarrow>^{+} .
\end{aligned}
$$

But, one can also construct the charge conjugation operator in the Fock space which acts, e.g., in the following way:

$$
\begin{aligned}
& \widetilde{U}_{[1 / 2]}^{c} a_{\uparrow}(\mathbf{p})\left(\widetilde{U}_{[1 / 2]}^{c}\right)^{-1}=-b_{\downarrow}(\mathbf{p}), \widetilde{U}_{[1 / 2]}^{c} a_{\downarrow}(\mathbf{p})\left(\widetilde{U}_{[1 / 2]}^{c}\right)^{-1}=-b_{\uparrow}(\mathbf{p}), \\
& \widetilde{U}_{[1 / 2]}^{c} b_{\uparrow}^{\dagger}(\mathbf{p})\left(\widetilde{U}_{[1 / 2]}^{c}\right)^{-1}=+a_{\downarrow}^{\dagger}(\mathbf{p}), \widetilde{U}_{[1 / 2]}^{c} b_{\downarrow}^{\dagger}(\mathbf{p})\left(\widetilde{U}_{[1 / 2]}^{c}\right)^{-1}=+a_{\uparrow}^{\dagger}(\mathbf{p}) .
\end{aligned}
$$


Therefore,

$$
\begin{aligned}
& \widetilde{U}_{[1 / 2]}^{c}\left|\mathbf{p}, \uparrow>^{+}=-\right| \mathbf{p}, \downarrow>^{-}, \widetilde{U}_{[1 / 2]}^{c}\left|\mathbf{p}, \downarrow>^{+}=-\right| \mathbf{p}, \uparrow>^{-} \\
& \widetilde{U}_{[1 / 2]}^{c}\left|\mathbf{p}, \uparrow>^{-}=+\right| \mathbf{p}, \downarrow>^{+}, \widetilde{U}_{[1 / 2]}^{c}\left|\mathbf{p}, \downarrow>^{-}=+\right| \mathbf{p}, \uparrow>^{+}
\end{aligned}
$$

Next, by straightforward verification one can convince ourselves about correctness of the assertions made in [11b] (see also [8]) that it is possible to have a situation when the operators of the space inversion and the charge conjugation commute each other in the Fock space. For instance,

$$
\begin{aligned}
& U_{[1 / 2]}^{c} U_{[1 / 2]}^{s}\left|\mathbf{p}, \uparrow>^{+}=+i U_{[1 / 2]}^{c}\right|-\mathbf{p}, \downarrow>^{+}=+i \mid-\mathbf{p}, \downarrow>^{-} \\
& U_{[1 / 2]}^{s} U_{[1 / 2]}^{c}\left|\mathbf{p}, \uparrow>^{+}=U_{[1 / 2]}^{s}\right| \mathbf{p}, \uparrow>^{-}=+i \mid-\mathbf{p}, \downarrow>^{-} .
\end{aligned}
$$

The second choice of the charge conjugation operator answers for the case when the $\widetilde{U}_{[1 / 2]}^{c}$ and $U_{[1 / 2]}^{s}$ operations anticommute:

$$
\begin{aligned}
& \widetilde{U}_{[1 / 2]}^{c} U_{[1 / 2]}^{s}\left|\mathbf{p}, \uparrow>^{+}=+i \widetilde{U}_{[1 / 2]}^{c}\right|-\mathbf{p}, \downarrow>^{+}=-i \mid-\mathbf{p}, \uparrow>^{-} \\
& U_{[1 / 2]}^{s} \widetilde{U}_{[1 / 2]}^{c}\left|\mathbf{p}, \uparrow>^{+}=-U_{[1 / 2]}^{s}\right| \mathbf{p}, \downarrow>^{-}=+i \mid-\mathbf{p}, \uparrow>^{-} .
\end{aligned}
$$

Finally, the time reversal anti-unitary operator in the Fock space should be defined in such a way that the formalism to be compatible with the CPT theorem. If we wish the Dirac states to transform as $V(T) \mid \mathbf{p}, \pm 1 / 2>=$ $\pm \mid-\mathbf{p}, \mp 1 / 2>$ we have to choose (within a phase factor), Refs. [18, 21]:

$$
S(T)=\left(\begin{array}{cc}
\Theta_{[1 / 2]} & 0 \\
0 & \Theta_{[1 / 2]}
\end{array}\right)
$$

Thus, in the first relevant case we obtain for the $\Psi\left(x^{\mu}\right)$ field, composed of $\lambda^{S, A}$ or $\rho^{A, S} 4$-spinors

$$
\begin{aligned}
& V^{T} a_{\uparrow}^{\dagger}(\mathbf{p})\left(V^{T}\right)^{-1}=a_{\downarrow}^{\dagger}(-\mathbf{p}), V^{T} a_{\downarrow}^{\dagger}(\mathbf{p})\left(V^{T}\right)^{-1}=-a_{\uparrow}^{\dagger}(-\mathbf{p}) \\
& V^{T} b_{\uparrow}(\mathbf{p})\left(V^{T}\right)^{-1}=b_{\downarrow}(-\mathbf{p}), V^{T} b_{\downarrow}(\mathbf{p})\left(V^{T}\right)^{-1}=-b_{\uparrow}(-\mathbf{p}),
\end{aligned}
$$

that is not surprising. 


\section{The Conclusions.}

Thus, we proceeded as in the textbooks and defined the parity matrix as $P=\gamma_{0}$, because the helicity 4-spinors satisfy the Dirac equation, and the 2nd-type $\lambda$-spinors satisfy the coupled Dirac equations. Nevertheless, the properties of the corresponding spinors appear to be different with respect to the parity both on the first and the second quantization level. This result is compatible with the statement made by Berestetskii, Lifshitz and Pitaevskii. We defined another charge conjugation operator in the Fock space, which also transforms the positive-energy 4 -spinors to the negative-energy ones. In this case the operators $P$ and $C$ commute (instead of the anticommutation in the Dirac case), that is related to the eigenvalues of the charge operator, as in the Foldy and Nigam paper.

\section{References}

[1] Z. K. Silagadze, On the internal parity of anti-particles. Sov. J. Nucl. Phys. 55 (1992) 392.

[2] V. V. Dvoeglazov, Helicity basis and parity. Int. J. Theor. Phys. 43 (2004) 1287.

[3] V. V. Dvoeglazov, Different quantum field constructs in the $(1 / 2,0) \oplus(0,1 / 2)$ representation. Mod. Phys. Lett. A12 (1997) 2741.

[4] D. V. Ahluwalia and M. Sawicki, Phys. Rev. D47 (1993) 5161, nucl-th/9603019; Phys. Lett. B335 (1994) 24, hep-th/9407095.

[5] D. V. Ahluwalia, M. B. Johnson and T. Goldman, Phys. Lett. B316 (1993) 102.

[6] V. V. Dvoeglazov, Int. J. Theor. Phys. 37 (1998) 1915, and references therein.

[7] I. M. Gel'fand and M. L. Tsetlin, Sov. Phys. JETP 4 (1957) 947; G. A. Sokolik, ibid. 6 (1958) 1170.

[8] B. P. Nigam and L. L. Foldy, Phys. Rev. 102 (1956) 1410.

[9] E. P. Wigner, in Group Theoretical Concepts and Methods in Elementary Particle Physics - Lectures of the Istanbul Summer School of Theoretical Physics, 1962, ed. F. Gürsey (Gordon and Breach, 1964). 
[10] A. Sankaranarayanan and R. H. Good, jr., Nuovo Cim. 36, (1965) 1303.

[11] N. D. S. Gupta, Nucl. Phys. B4 (1967) 147; D. V. Ahluwalia, Int. J. Mod. Phys. A11 (1996) 1855; V. Dvoeglazov, Hadronic J. 20 (1997) 435.

[12] G. Ziino, Ann. Fond. Broglie 14 (1989) 427; ibid 16 (1991) 343; A. Barut and G. Ziino, Mod. Phys. Lett. A8 (1993) 1011; G. Ziino, Int. J. Mod. Phys. A11 (1996) 2081.

[13] M. Markov, ZhETF 7 (1937) 579; ibid., p. 603; Nucl. Phys. 55 (1964) 130.

[14] Yu. V. Novozhilov, Introduction to Elementary Particle Physics (Pergamon, 1975) $\S 4.3,6.2$.

[15] M. Jackob and G. C. Wick, Ann. Phys. 7 (1959) 404.

[16] D. A. Varshalovich, A. N. Moskalev and V. K. Khersonskiü, Quantum Theory of Angular Momentum (World Scientific, 1988), §6.2.5.

[17] V. V. Dvoeglazov, Fizika B6 (1997) 111.

[18] C. Itzykson and J.-B. Zuber, Quantum Field Theory (McGraw-Hill Book Co., 1980), Ch. 3-4.

[19] V. B. Berestetskiı̌, E. M. Lifshitz and L. P. Pitaevskiŭ, Quantum Electrodynamics (Pergamon Press, 1982), §16.

[20] W. Greiner, Field Quantization (Springer, 1996), Ch. 10.

[21] N. N. Bogoliubov and D. V. Shirkov, Introduction to the Theory of Quantized Fields (Wiley, 1980).

[22] S. Weinberg, The Quantum Theory of Fields. Vol. I. Foundations. (Cambridge UP, 1995). 